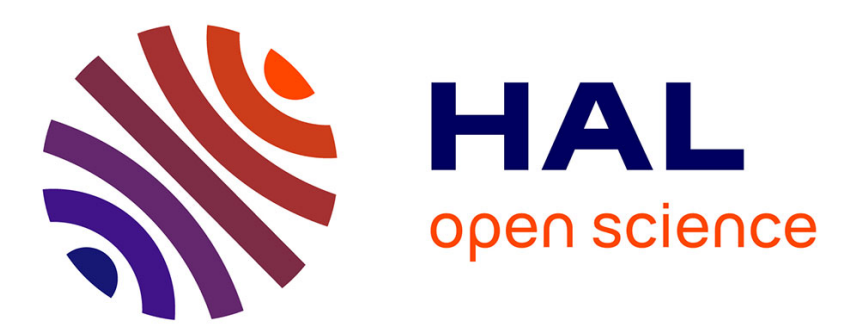

\title{
A kinetic model for particle-surface interaction applied to rain falling on water waves
}

\author{
Fabrice Veron, Luc Mieussens
}

\section{To cite this version:}

Fabrice Veron, Luc Mieussens. A kinetic model for particle-surface interaction applied to rain falling on water waves. Journal of Fluid Mechanics, 2016, 796, pp.767-787. 10.1017/jfm.2016.252 . hal$01245956 \mathrm{v} 2$

\section{HAL Id: hal-01245956 \\ https://hal.science/hal-01245956v2}

Submitted on 25 Nov 2016

HAL is a multi-disciplinary open access archive for the deposit and dissemination of scientific research documents, whether they are published or not. The documents may come from teaching and research institutions in France or abroad, or from public or private research centers.
L'archive ouverte pluridisciplinaire HAL, est destinée au dépôt et à la diffusion de documents scientifiques de niveau recherche, publiés ou non, émanant des établissements d'enseignement et de recherche français ou étrangers, des laboratoires publics ou privés. 


\title{
A kinetic model for particle-surface interaction applied to rain falling on water waves
}

\author{
Fabrice Veron $^{1} \dagger$ and Luc Mieussens ${ }^{2}$ \\ ${ }^{1}$ School of Marine Science and Policy, University of Delaware, Newark DE, USA \\ ${ }^{2}$ Univ. Bordeaux, IMB, UMR 5251, F-33400 Talence, France.
}

(Received $\mathrm{xx}$; revised $\mathrm{xx}$; accepted $\mathrm{xx}$ )

We present a model for estimating the momentum flux from water drops falling onto a moving free surface. The theory is based on a kinetic approach whereby individual drops are modelled as point particles with mass and velocity, and are described collectively by a distribution function $f(t, \boldsymbol{x}, \boldsymbol{v}, r)$. We show that the resulting momentum flux can be readily incorporated in free surface Navier-Stokes and Euler models. As an illustration of this approach we examine the interaction between rainfall and linear deep water surface waves. This particular application is not fundamentally different from study of Le Méhauté \& Khangaonkar (1990), but our methodology is more general and is novel in its use of a kinetic approach with an all-purpose drop distribution function. The applicability of the model to linear surface waves is found to be valid for surface wave wavelengths approximately ranging from $3 \mathrm{~m}$ to $250 \mathrm{~m}$. We further show that rainfall modifies the usual wave dispersion relationship and induces wave amplification, or damping, depending on the rain rate, the rain impact angle, and the wavelength of the surface wave. We solve for the amplification and damping rates analytically and show, among other results, that rain falling vertically will always damp the surface waves.

\section{Introduction}

Rain falling on the sea surface and, in particular, the observed damping of the surface waves by rainfall, is a problem that has puzzled the sea going and scientific communities alike. Indeed, sailors have long noted that rainfall has the unexpected effect of "calming the sea". Among the scientific community, this observation was first made by Reynolds (1900) who described surface wave damping in the presence of rain. Interestingly, this rain-induced wave damping may lead to biases and errors in remote wind and wave measurements routinely made by airborne microwave radiometers which rely on backscatter returns from the surface waves (Chen et al. 1998). Indeed, while raindrops in the atmosphere reduce overall backscatter intensity, an effect that has been used to locate patches of rainfall in the open ocean (Atlas 1994; Chen et al. 1998; Bliven et al. 1993; Braun et al. 2002; Contreras et al. 2003; Weissman et al. 2012), reduced surface roughness, or wave damping, has the opposite effect and instead enhances the radar backscatter signal (Guymer et al. 1995; Quartly et al. 1996). Thus, in order to improve the satellite measurements in rainy conditions, a physical understanding of raininduced wave damping mechanism is necessary if adequate parametrizations are to be developed.

In addition, it is well known that the surface waves play a critical role in the air-sea

$\dagger$ Email address for correspondence: fveron@udel.edu 
momentum flux and air-sea drag. An accurate knowledge of surface wave amplitude and slope is thus necessary in order to make reliable wind speed predictions, especially in high wind, rainy conditions. Indeed, the role of surface waves and associated phenomena (e.g. breaking, sea spray, wave stress, Langmuir turbulence) on the air-sea fluxes have been well documented and studied as an important component of the air-sea surface dynamics, with significant progress achieved in the past decades (Melville 1996; Thorpe 2004; Sullivan \& McWilliams 2010; Veron 2015). However, the dynamic effects of rainfall have been largely overlooked and, in particular, our knowledge of the interaction between rain and surface wave remains extremely poor (Kraus \& Bussinger 1994; Jones \& Toba 2001; Csanady 2001).

To date, relatively few experimental and theoretical investigations of rain-wave interactions have been completed. The available experimental data are, for the most part, focused on quantifying the rain-induced wave damping in a laboratory environment, where waves are shorter than approximately $0.5 \mathrm{~m}$ in wavelength (Manton 1973; Tsimplis \& Thorpe 1989; Tsimplis 1992; Poon et al. 1992; Yang et al. 1997; Braun et al. 2002; Harrison 2012; Peirson et al. 2013). Some of these experiments were performed using mechanically generated, monochromatic waves (Tsimplis \& Thorpe 1989), while others used wind-generated wave fields (Poon et al. 1992). In both types of experiments, gravity waves with frequencies less than $5 \mathrm{~Hz}$ were damped by the rain and the damping rate was found to be independent of the rain rate. Wave amplification has also been observed for higher wave frequencies (Poon et al. 1992; Yang et al. 1997; Braun et al. 2002; Harrison 2012) but only in the presence of wind.

Tsimplis \& Thorpe (1989) proposed that the observed rain-induced wave damping results from the rain-generated turbulence where a near-surface high eddy viscosity layer viscously damps the surface waves. Shortly after, Nystuen (1990) estimated the wave attenuation when a thin, rain-induced, high viscosity mixed layer is present near the surface. There have been, however, very few experimental studies focusing on direct raininduced turbulence measurements (Braun 2003; Beya et al. 2011; Harrison 2012; Peirson et al. 2013). Evidently, since the rain-induced turbulent layer is $O(10) \mathrm{cm}$ thick (Green \& Houk 1979b; Harrison 2012; Peirson et al. 2013), viscous damping will only affect surface waves shorter than $O(1) \mathrm{m}$ such as those observed in the laboratory (Nystuen 1990). The rain-induced damping of longer ocean waves documented by Chen et al. (1998) for example, likely results from a different mechanism.

Le Méhauté \& Khangaonkar (1990) modelled rain-wave interactions based only on the rain drop's momentum change at the air-sea interface. Their theory suggests several rain-wave interaction regimes, with a switch from damping to enhancing wave amplitude depending on the entry angle of the rain drops. At high wind speed (high entry angle of rain drops), Le Méhauté \& Khangaonkar (1990) predicted that the rain will increase the waves' amplitude while at low wind speed (vertical rain), the rain causes significant wave damping, especially at high wave frequencies (short waves). In that case, the damping rate was found to increase linearly with rain rate. In their study Le Méhauté \& Khangaonkar (1990) did not account for a distribution of rain drop size or fall velocities, nor did they provide details on the range of conditions for which the theory might not be valid. The data of both Poon et al. (1992) and Tsimplis (1992) show damping rates that are independent of rain rates and approximately 10 times larger than those predicted by the theory of Le Méhauté \& Khangaonkar (1990). As emphasized by Peirson et al. (2013), this discrepancy has yet to be resolved. To date, the damping of the surface by rainfall has not been satisfactorily explained and available theories remain untested.

Simple estimates show that shear stresses (momentum flux) induced by rainfall at the surface under typical rainfall conditions can be as significant, dynamically, as the 
wind induced stresses (Caldwell \& Elliott 1971, 1972). Thus, as a first step, we propose a model based on surface momentum exchange induced by the rain. In this paper, our approach uses a kinetic description of rain where individual drops are modelled as point particles with mass and velocity. Collectively, they are described by a velocity and mass (or, equivalently, velocity and size) distribution. This idea is standard in kinetic theory of gases (Chapman \& Cowling 1990), and it is valid under some assumptions like, for instance, when the scale of the drop is much smaller than the macroscopic length scales of interest. This theory has been used successfully in other fields, such as in spray combustion modelling (see Marchisio \& Fox 2013). To the best of our knowledge, this is the first time that kinetic theory is applied to the natural rainfall - free surface interaction problem.

The kinetic approach is a much richer description than the momentum flux approach of Le Méhauté \& Khangaonkar (1990) in that it allows to consider rainfall with a polydisperse distribution, that is a distribution of drop radii, and radius-dependent impact velocities and impact angles. This added physical complexity makes the kinetic approach substantially more realistic. Other drop properties like temperature, density, or chemical composition could also be easily incorporated making this approach more powerful than the standard rain rate, or size distribution, methodology (e.g Law \& Parsons 1943; Marshall \& Palmer 1948; Green \& Houk 1979b; Schlussel et al. 1997; de Wolf 2001). Finally, it allows for accurate estimation of the stress exerted by the rain on the sea surface. This stress takes into account the velocity of the drops with respect to the movement of the surface, and we show that it can be readily incorporated into free surface fluid models using Navier-Stokes or Euler frameworks.

This article is organized as follows: In section 2, we explain how rain drops can be modeled at the kinetic level (section 2.1). Then, we compute the rain stress induced at the sea surface (section 2.2), and we show how it can be taken into account in free surface Navier-Stokes and Euler models (section 2.3). An Euler model is then employed in section 3 to develop a linear water wave analysis with falling rain. A specific rain drop distribution is then considered and utilized in sections 3.1 and 3.2. The validity regime of this analysis is carefully explored in section 3.3, and some numerical results are presented (section 4). Finally, we offer a discussion (section 5) followed by brief conclusions in section 6 .

\section{Free surface Navier-Stokes and Euler models with falling rain}

\subsection{Kinetic description of the rain}

The kinetic description employed here can be used to compute the force, and hence the pressure, exerted by drops falling on a surface (see Chapman \& Cowling 1990). For the sake of completeness, and since the reader might not be familiar with kinetic gas theory, we find it useful to briefly summarize below certain aspects of our approach.

A rain drop is a volume of fluid described by its density and velocity, which are in turn governed by the Navier-Stokes equations. At larger scales, the number of drops falling on a unit surface at the air-sea interface is large, and conversely, the radius of any drop is much smaller than the macroscopic length scales (e.g. the surface gravity waves). Thus, it is appropriate to use a statistical description of the drops. This description is similar to that used for spray modeling, as is commonly done in combustion engineering (for example see Marchisio \& Fox (2013)).

We assume that each rain drop is made of a single fluid with constant density $\rho_{d}$, and 


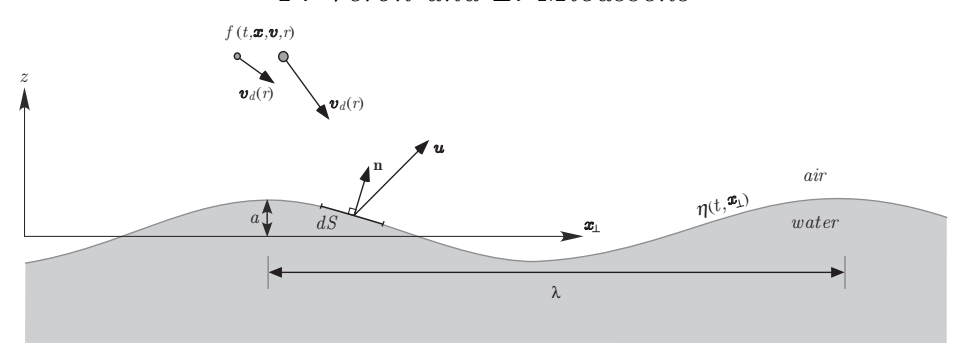

FIGURE 1. Schematic, notation and sign convention for rain drops falling on the sea surface.

is spherical with radius $r$ and mass $m(r)=\rho_{d} \frac{4}{3} \pi r^{3}$. Each drop is then uniquely defined by:

- its position $\boldsymbol{x} \in \mathbb{R}^{3}$;

- its velocity $\boldsymbol{v} \in \mathbb{R}^{3}$, which is the mean velocity of the molecules inside the drop;

- and its radius $r \in] 0,+\infty[$.

One could also consider additional variables such as temperature or chemical species but this will not be used here.

The set of drops is then described by the distribution function $f(t, \boldsymbol{x}, \boldsymbol{v}, r)$, which is the number density of drops at time $t$ in the phase space $\left.\mathbb{R}^{3} \times \mathbb{R}^{3} \times\right] 0,+\infty[$. In other words, $f(t, \boldsymbol{x}, \boldsymbol{v}, r) d \boldsymbol{x} d \boldsymbol{v} d r$ is the number of drops that at time $t$ are at position $\boldsymbol{x} \pm d \boldsymbol{x}$ (where $d \boldsymbol{x}=d x d y d z$ ) with velocity $\boldsymbol{v} \pm d \boldsymbol{v}$ and with a radius $r \pm d r$.

Averaged quantities can be easily obtained through the distribution $f(t, \boldsymbol{x}, \boldsymbol{v}, r)$. For instance, the time-dependent radius distribution $n$ is

$$
n(t, \boldsymbol{x}, r)=\int_{\mathbb{R}^{3}} f(t, \boldsymbol{x}, \boldsymbol{v}, r) d \boldsymbol{v},
$$

which is the number of drops of radius $r$ at time $t$ and position $\boldsymbol{x}$, per unit volume and per radius increment.

\subsection{Pressure induced by drops on a surface}

To compute the surface stress exerted by rain drops, we first consider a surface $d S$ defined by a normal unit vector $\mathbf{n}$ and moving with a velocity $\boldsymbol{u}$; we trust the reader will not to confuse the unit vector $\mathbf{n}$ with the drop size distribution $n$ (equation 2.1). Here, we are interested in the drops with a given velocity $\boldsymbol{v}$ that will impact $d S$. This simply implies that we only need to consider drops for which $(\boldsymbol{v}-\boldsymbol{u}) \cdot \mathbf{n}<0$ (see figure 1). Thus, summing over all possible radii and all incident velocities, the total number of drops impacting $d S$ during a short time $d t$ is given by:

$$
N=\left(\int_{(\boldsymbol{v}-\boldsymbol{u}) \cdot \mathbf{n}<0} \int_{] 0,+\infty[} f(t, \boldsymbol{x}, \boldsymbol{v}, r)(-(\boldsymbol{v}-\boldsymbol{u}) \cdot \mathbf{n}) d r d \boldsymbol{v}\right) d S d t .
$$

In the frame of reference attached to the surface, the momentum from all the drops impacting $d S$ is

$$
\boldsymbol{M}=\left(\int_{(\boldsymbol{v}-\boldsymbol{u}) \cdot \mathbf{n}<0} \int_{] 0,+\infty[} m(r)(\boldsymbol{v}-\boldsymbol{u}) f(t, \boldsymbol{x}, \boldsymbol{v}, r)(-(\boldsymbol{v}-\boldsymbol{u}) \cdot \mathbf{n}) d r d \boldsymbol{v}\right) d S d t .
$$

Consequently, the momentum flux $\varphi$ (the momentum per unit of surface and per unit of 
time) across a surface of normal $\mathbf{n}$ is obtained by dividing $\boldsymbol{M}$ by $d S d t$ such that:

$$
\begin{aligned}
\boldsymbol{\varphi} & =\int_{(\boldsymbol{v}-\boldsymbol{u}) \cdot \mathbf{n}<0} \int_{] 0,+\infty[} m(r)(\boldsymbol{v}-\boldsymbol{u}) f(t, \boldsymbol{x}, \boldsymbol{v}, r)(-(\boldsymbol{v}-\boldsymbol{u}) \cdot \mathbf{n}) d r d \boldsymbol{v} \\
& =-\left(\int_{(\boldsymbol{v}-\boldsymbol{u}) \cdot \mathbf{n}<0} \int_{] 0,+\infty[} m(r)(\boldsymbol{v}-\boldsymbol{u}) \otimes(\boldsymbol{v}-\boldsymbol{u}) f(t, \boldsymbol{x}, \boldsymbol{v}, r) d r d \boldsymbol{v}\right) \mathbf{n}
\end{aligned}
$$

where $\otimes$ denotes the tensor product. Therefore, the force exerted by the rain drops on the surface $d S$ is

$$
\boldsymbol{F}=\varphi d S,
$$

where $\varphi$ is the momentum flux of the drops defined in equation (2.4). Here, we neglect the force exerted by the drops ejected from the surface. When rain falls on a free surface, there are indeed secondary drops generated at the surface. Drops pinch off from the stalks, and smaller so-called film droplets are ejected from crowns and craters resulting from rain-drop impacts. However, these secondary drops are either much slower than the rain in the case of stalks, or significantly smaller than rain drops in the case of film droplets (see Veron 2015). In either case, the momentum carried by these secondary drops is considered negligible.

We finally consider that drops falling on the surface exert a stress on $d S$ such that the corresponding stress tensor $\boldsymbol{\sigma}_{d}$ is given by the relation $\boldsymbol{F}=\boldsymbol{\sigma}_{d} \mathbf{n} d S$. Using equations (2.4) and (2.5), this analysis then yields:

$$
\boldsymbol{\sigma}_{d}=-\int_{(\boldsymbol{v}-\boldsymbol{u}) \cdot \mathbf{n}<0} \int_{] 0,+\infty[} m(r)(\boldsymbol{v}-\boldsymbol{u}) \otimes(\boldsymbol{v}-\boldsymbol{u}) f(t, \boldsymbol{x}, \boldsymbol{v}, r) d r d \boldsymbol{v}
$$

\subsection{Accounting for falling rain in Navier-Stokes and Euler free-surface models}

In this section, we incorporate the rain-induced stress (equation (2.6) above) in an incompressible Navier-Stokes framework which includes a free surface. The surface is described by the equation $z=\eta\left(t, \boldsymbol{x}_{\perp}\right)$, where $t>0$ is the time, $\boldsymbol{x}_{\perp}=(x, y)$ is the two-dimensional horizontal position vector perpendicular to the $z$ direction, and $\eta$ is the height of the free surface (see figure 1). The velocity $\boldsymbol{u}\left(t, \boldsymbol{x}_{\perp}, z\right)=\left(\boldsymbol{u}_{\perp}, w\right)$ of the water under the free surface is given by

$$
\begin{aligned}
& \nabla \cdot \boldsymbol{u}=0, \\
& \rho \partial_{t} \boldsymbol{u}+\rho(\boldsymbol{u} \cdot \nabla) \boldsymbol{u}=\nabla \cdot \boldsymbol{\sigma}+\rho \boldsymbol{g},
\end{aligned}
$$

where $\boldsymbol{\sigma}=-p \boldsymbol{I}+\mu\left(\nabla \boldsymbol{u}+(\nabla \boldsymbol{u})^{T}\right)$ is the stress tensor, $\rho$ is the water density, $p$ is the pressure, $\mu$ is the dynamic viscosity of water, and $\boldsymbol{g}$ is gravity. Here, $\boldsymbol{I}$ is the unit tensor, $\nabla \boldsymbol{u}$ is the gradient velocity tensor and $(\nabla \boldsymbol{u})^{T}$ its transpose. The boundary conditions at the free surface are the kinematic boundary condition

$$
w\left(t, \boldsymbol{x}_{\perp}, z=\eta\left(t, \boldsymbol{x}_{\perp}\right)\right)=\partial_{t} \eta+\boldsymbol{u}_{\perp} \cdot \nabla_{\perp} \eta,
$$

and the dynamic boundary condition

$$
\boldsymbol{\sigma} \mathbf{n}=\boldsymbol{\sigma}_{a} \mathbf{n}+\Gamma \kappa \mathbf{n},
$$

where $\boldsymbol{\sigma}_{a}$ is the stress tensor exerted by the air on thec surface, $\Gamma$ is the surface tension, and $\kappa$ is the curvature of the free surface. In the absence of wind and other shear stresses, the stress tensor reduces to $\boldsymbol{\sigma}_{a}=-p_{a} \boldsymbol{I}$, where $p_{a}$ is the atmospheric pressure.

Therefore, we can account for the effect of the rain drops on the free surface by simply incorporating the stress from the rain (2.6) into the dynamic boundary condition (2.10), 
which is then replaced by

$$
\boldsymbol{\sigma} \mathbf{n}=\boldsymbol{\sigma}_{a} \mathbf{n}+\Gamma \kappa \mathbf{n}+\boldsymbol{\sigma}_{d} \mathbf{n} .
$$

Neglecting viscosity both in the fluid and at the surface, and neglecting surface tension (thus limiting our analysis to moderate surface curvatures), we obtain the following Euler model:

$$
\begin{aligned}
& \nabla \cdot \boldsymbol{u}=0, \\
& \rho \partial_{t} \boldsymbol{u}+\rho(\boldsymbol{u} \cdot \nabla) \boldsymbol{u}+\nabla p=\rho \boldsymbol{g},
\end{aligned}
$$

with the unchanged kinematic relation (2.9). Since viscosity is neglected, the dynamic boundary condition is limited to normal stresses only and reduces to:

$$
p=p_{a}+p_{d}
$$

where $p_{a}$ is the atmospheric pressure and $p_{d}=-\boldsymbol{\sigma}_{d} \mathbf{n} \cdot \mathbf{n}$ is the pressure exerted by the rain on the free surface expressed as:

$$
p_{d}=\int_{(\boldsymbol{v}-\boldsymbol{u}) \cdot \mathbf{n}<0} \int_{] 0,+\infty[} m(r)|(\boldsymbol{v}-\boldsymbol{u}) \cdot \mathbf{n}|^{2} f(t, \boldsymbol{x}, \boldsymbol{v}, r) d r d \boldsymbol{v} .
$$

\section{Application to water waves with falling rain}

\subsection{Linear deep water surface gravity waves with falling rain}

Here, we use the standard linear water wave theory using the inviscid Euler model (equations (2.12)-(2.14)). For an irrotational flow, the linearized Euler system written in the potential form, gives

$$
\begin{aligned}
& \nabla^{2} \Phi=0, \\
& \rho \partial_{t} \Phi+p+\rho g z=C(t),
\end{aligned}
$$

where $\Phi$ is the velocity potential such that $\boldsymbol{u}=\nabla \Phi$ is the velocity associated with the surface waves, and $C(t)$ depends only on time $t$. The surface boundary conditions (2.9) and (2.14) give

$$
\begin{aligned}
& \partial_{t} \eta=\partial_{z} \Phi, \\
& p=p_{a}+p_{d} .
\end{aligned}
$$

Finally, we consider that velocity perturbations from the surface waves are confined near the surface (deep water waves) and add the kinematic boundary condition

$$
\Phi=0 \text { as } z \rightarrow-\infty
$$

Considering a surface wave mode of elevation $\eta=a \mathrm{e}^{\mathrm{i}\left(\boldsymbol{k}_{\perp} \cdot \boldsymbol{x}_{\perp}-\omega t\right)}$ with amplitude $a$, angular frequency $\omega$, and propagating along the horizontal wavenumber $\boldsymbol{k}_{\perp}$, the Laplace equation (3.1) and accompanying linearized kinematic conditions (3.3) and (3.5) give $\Phi=-\mathrm{i} \frac{a \omega}{k} e^{k z} \mathrm{e}^{\mathrm{i}\left(\boldsymbol{k}_{\perp} \cdot \boldsymbol{x}_{\perp}-\omega t\right)}$ with $k=\left|\boldsymbol{k}_{\perp}\right|$ and the surface wave wavelength $\lambda=k /(2 \pi)$. Note that the linear theory assumes that $a k$ is a small parameter. In turn, Bernouilli's equation (3.2) and the dynamic condition (3.4) linearized about $z=0$ lead to the usual dispersion relationship $\omega=(g k)^{1 / 2}$ for deep water surface gravity waves when $p_{d}=0$ and choosing $C(t)=p_{a}$. Therefore, we can now examine how incorporating the raininduced pressure in the dynamic boundary condition modifies the classical dispersion relationship.

In order to do so, the rain-induced pressure defined in equation (2.15) must be evaluated. To make the computation possible, we make several assumptions. First, we 
assume the rain drop distribution is homogeneous in space and monokinetic, that is to say $f(t, \boldsymbol{x}, \boldsymbol{v}, r)=n(r) \delta_{\boldsymbol{v}-\boldsymbol{v}_{d}(r)}$, where $\delta$ is the delta-Dirac function centered on $\boldsymbol{v}_{d}$ so that $\delta_{\boldsymbol{v}-\boldsymbol{v}_{d}(r)}=0$ for $\boldsymbol{v} \neq \boldsymbol{v}_{d}$. In other words, we assume the same drop radius distribution $n(r)$ at each time $t$ and position $\boldsymbol{x}$, and all the drops of radius $r$ have the same velocity $\boldsymbol{v}_{d}(r)$. Note that despite its simplicity, this choice allows for different drop velocities depending on the radius. Indeed, various analytic formulae of radius distribution and velocity can be used (see section 3.2). Second, we estimate the integral (2.15) assuming that $\left(\boldsymbol{v}_{d}(r)-\boldsymbol{u}\right) \cdot \mathbf{n}<0$ for all drop radii. With these assumptions, the rain-induced pressure (equation $(2.15)$ ) reduces to

$$
p_{d}=\int_{] 0,+\infty[} m(r) n(r)\left|\left(\boldsymbol{v}_{d}(r)-\boldsymbol{u}\right) \cdot \mathbf{n}\right|^{2} d r .
$$

This second assumption simply means that, in the estimate of the rain-induced pressure, we count all drop contributions including very slow falling drops for which $\left(\boldsymbol{v}_{d}(r)-\right.$ $\boldsymbol{u}) \cdot \mathbf{n}$ may not be negative. In other words, we include drops of very small radii that may locally fall slower than the wavy surface. We will show in the following section that these small droplets (as is the case with secondary rain-generated drops) carry a negligible fraction of the total rain-induced momentum flux. However, their inclusion here significantly simplifies the integration (3.6) and yields practical analytical expressions (see appendix A). Note here that $p_{d}$ is quadratic with the impact velocity, and cubic with the drop radius.

Without loss of generality, we choose a frame of reference aligned with $\boldsymbol{x}_{\perp}$ (i.e. such that $\boldsymbol{x}_{\perp}=(x, 0)$ is the direction of propagation of the wave) and project $\boldsymbol{v}_{d}(r)$ with the horizontal and vertical components of the drop velocity given by $\boldsymbol{v}_{d}(r)=\left(u_{d}(r), w_{d}(r)\right)$ respectively. Keeping only the linear terms in $a k$ and $a \omega$, the rain-induced pressure $p_{d}$ from equation (3.6) reduces to $p_{d}=p_{d}^{(0)}+p_{d}^{(1)}$ with

$$
\begin{aligned}
& p_{d}^{(0)}=\int_{] 0,+\infty[} m(r) n(r) w_{d}(r)^{2} d r, \\
& p_{d}^{(1)}=(I k+J \omega) \mathrm{i} a \mathrm{e}^{\mathrm{i}(k x-\omega t)},
\end{aligned}
$$

and where $I$ and $J$ are constants defined by

$$
\begin{aligned}
& I=-2 \int_{] 0,+\infty[} m(r) n(r) u_{d}(r) w_{d}(r) d r \\
& J=2 \int_{] 0,+\infty[} m(r) n(r) w_{d}(r) d r .
\end{aligned}
$$

Here, $p^{(0)}$ is a constant pressure term (thus dynamically insignificant) arising from the vertical momentum flux due to the rain. Also, $p^{(1)}$ is a wave-coherent pressure component in which the term Iak results from the surface slope - drop velocity correlation and the term $J a \omega$ is caused by the vertical velocity of the interface. We note here that $J /\left(2 \rho_{d}\right)$ is the rain-induced volume flux per horizontal (i.e. perpendicular to $w_{d}$ ) unit surface per unit time. It has the dimension of a velocity and is commonly referred to as the rain rate $R$. We also note that with our sign convention where the vertical $z$-direction is positive upward, $w_{d}<0$ and $J /\left(2 \rho_{d}\right)=R$ where $R<0$. Generally, rain rates are reported in mm $\mathrm{h}^{-1}$ and refer to $|R|$.

We now re-introduce the dynamic condition (3.4) with $p_{d}=p_{d}^{(0)}+p_{d}^{(1)}$ given by equations (3.7) - (3.8) into Bernouilli's equation (3.2) linearized about $z=0$ and obtain

$$
\rho \partial_{t} \Phi+p_{d}^{(1)}+\rho g \eta=0
$$


where we have chosen the constant $C(t)$ such that $C(t)=p_{a}+p_{d}^{(0)}$. Inserting the expressions of $\Phi, p_{d}^{(1)}$, and $\eta$ into this relation gives the following equation for $\omega$ :

$$
\omega^{2}-\mathrm{i} \frac{k J}{\rho} \omega-\left(g+\mathrm{i} \frac{k I}{\rho}\right) k=0 .
$$

In this case, we find that the dispersion relationship is a quadratic equation with complex coefficients. We find that its solutions are couples of complex frequencies $\omega=\omega_{R}+\mathrm{i} \omega_{I}$. The real part $\omega_{R}$ is given by

$$
\omega_{R}= \pm \sqrt{\frac{1}{2}\left(\sqrt{\left(k g-\frac{k^{2} J^{2}}{4 \rho^{2}}\right)^{2}+\frac{k^{4} I^{2}}{\rho^{2}}}+k g-\frac{k^{2} J^{2}}{4 \rho^{2}}\right)} .
$$

Without loss of generality, we choose the solution with $\omega_{R}>0$ (surface waves going in the $x>0$ direction), and we find that the imaginary part $\omega_{I}$ depends on the sign of $I$ :

$$
\omega_{I}=\frac{k J}{2 \rho}+\operatorname{sign}(I) \sqrt{\frac{1}{2}\left(\sqrt{\left(k g-\frac{k^{2} J^{2}}{4 \rho^{2}}\right)^{2}+\frac{k^{4} I^{2}}{\rho^{2}}}-k g+\frac{k^{2} J^{2}}{4 \rho^{2}}\right)}
$$

where we consider that $\operatorname{sign}(0)=0$. Since $\eta=a \mathrm{e}^{\mathrm{i}(k x-\omega t)}=a \mathrm{e}^{\omega_{I} t} \mathrm{e}^{\mathrm{i}\left(k x-\omega_{R} t\right)}$, the falling rain induces wave attenuation if $\omega_{I}<0$, or amplification if $\omega_{I}>0$.

\subsection{Rain size and velocity distributions}

In order to assess the impact of rainfall on the surface waves, we must evaluate equations (3.11) and (3.12) and thus estimate $I$ and $J$. To do so, we need to choose a number density function $n(r)$ and a fall velocity $\boldsymbol{v}_{d}(r)$ for the rain. There are number of different formulae for either $n(r)$ or $\boldsymbol{v}_{d}(r)$ in the literature but we note here that $I$ and $J$ depend on their integral values and are thus not dramatically influenced by the details of their formulations. For the drop size distribution, we choose the classical gamma function formulation proposed by Marshall \& Palmer (1948):

$$
n(r)=\mathcal{N} \mathrm{e}^{-\Lambda r},
$$

where $\Lambda=344.34|R|^{-0.21} \mathrm{~m}^{-1}$ with $R$ in $\mathrm{m} \mathrm{s}^{-1}$, and $\mathcal{N}$ is a normalization factor (see appendix A) that allows to recover the rain rate which is the variable generally measured and reported. The vertical fall velocity $w_{d}(r)$ is taken from Best (1950):

$$
w_{d}(r)=-\Upsilon_{w}\left(1-\mathrm{e}^{-\alpha_{w} r}\right),
$$

with $\Upsilon_{w}=9.32 \mathrm{~m} \mathrm{~s}^{-1}$ the maximum fall velocity for rain, and $\alpha_{w}=1296 \mathrm{~m}^{-1}$. Equation (3.14) is considered valid for drop sizes $0.1 \mathrm{~mm}<r<3 \mathrm{~mm}$. The horizontal rain velocity is usually ignored (Ho et al. 2000; Harrison et al. 2012) or considered to be independent of the radius and assumed to be approximately $85 \%$ of the $10-\mathrm{m}$ wind speed $U_{10}$ (Caldwell \& Elliott 1971, 1972). However, while it is clear that large rain drops falling through the near surface atmospheric boundary layer will rapidly reach the surface without having the time to decelerate significantly, small droplets take longer to fall and also have a smaller mass and Stokes number (inertial response time). Therefore, the small rain drops will impact the surface after having exchanged momentum with the air flow and thus decelerated substantially. Past studies have focused on the vertical terminal velocity of rain, and there are few published studies on the horizontal transport of rain drops. To the authors' knowledge, there are no published radius-dependent horizontal drop velocity 

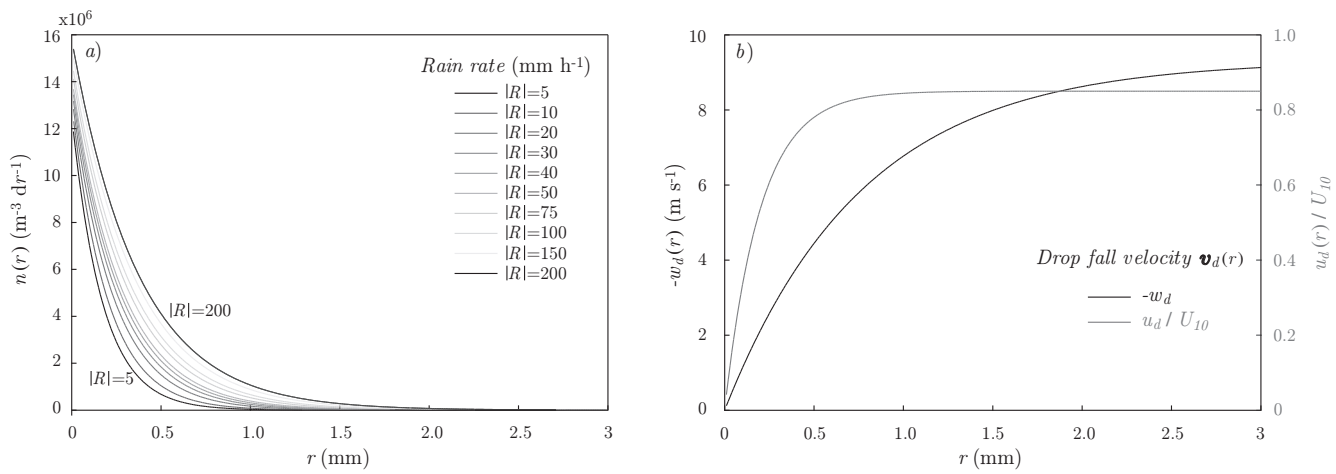

Figure 2. a) The rain drop size distribution from Marshall \& Palmer (1948) for multiple rain rates. b) the vertical drop velocity $w_{d}$, and horizontal velocity $u_{d}$ normalized by $U_{10}$ (right axis) at impact on the surface, as a function of drop radius.

formulae. To remedy this paucity, we used results from a series of simulations based on the Lagrangian Stochastic model of Mueller \& Veron (2009) for wind speeds ranging from $0 \mathrm{~m} \mathrm{~s}^{-1}$ to $30 \mathrm{~m} \mathrm{~s}^{-1}$. In these simulations, rain drops of various sizes were left to fall freely through the turbulent atmospheric boundary layer above the wavy surface until they impacted the surface. Impact velocities were recorded and based on the results from these simulations $\dagger$, we propose the following formulation for rain drop horizontal velocity at impact on the surface:

$$
\left.u_{d}(r)\right|_{z=0}=\Upsilon_{u}\left(1-\mathrm{e}^{-\alpha_{u} r}\right)
$$

with $\Upsilon_{u}=0.85 U_{10}$ and $\alpha_{u} \approx 5000 \mathrm{~m}^{-1}$. Note that equation 3.15 implies that the wind and wave are traveling in the same $x$ direction. In the case of misaligned wind and waves, $u_{d}$ is simply the component of the horizontal drop velocity in the direction of propagation of the wave and $\Upsilon_{u}=0.85 U_{10} \cos \theta$, with $\theta$ the angle between the wind and the wave directions.

Figure 2a shows the drop size distribution of Marshall \& Palmer (1948) for several rain rates. As expected the number density increases with increasing rain rate but the shape of the distribution remains. Figure $2 \mathrm{~b}$ shows the rain drop velocity at impact on the surface as a function of the radius. The vertical fall velocity is that of Best (1950) given by equation (3.14). The horizontal drop impact velocity, shown in gray, is normalized by the $10-\mathrm{m}$ wind speed $U_{10}$. Large drops are found to impact at approximately $85 \%$ of the 10-m wind speed in accord with Caldwell \& Elliott (1972).

\subsection{Validity of assumptions}

In this section, we discuss the validity of several assumptions made above and offer limits on the range of validity of the approach presented here. Overall, we show below that the model presented here is valid for linear deep water gravity waves with wavelength in the range $O(1-3) \mathrm{m}<\lambda<O(250) \mathrm{m}$.

\subsubsection{Inviscid irrotational approach}

As mentioned earlier, rainfall is known to generate a near surface highly turbulent and dissipative layer that is $O(10) \mathrm{cm}$ (Braun 2003; Beya et al. 2011; Harrison 2012; Peirson

$\dagger$ These simulations explore the free fall of water drops of several sizes through the stratified turbulent atmosphere near the wavy interface. They are very comprehensive and thus beyond the scope of this paper. Results will be the subject of a subsequent publication. 
et al. 2013). Hence, surface waves with wavelength significantly longer than the depth of this layer will be largely unaffected, and conversely, the viscous damping mechanism proposed by Tsimplis \& Thorpe (1989) will only affect waves with wavelength shorter than $O(1) \mathrm{m}$ such as those observed in the laboratory. However, the analysis presented above for application to water waves (section 3.1), is limited to inviscid irrotational flows. Thus, the model presented here, in which the effects of the rain reduce to an added pressure confined to the interface, does not account for the dissipation from the rain-generated subsurface turbulent layer. This merely implies that the present approach is suitable for evaluating rain-induced effects for waves with wavelength $\lambda$ longer than $O(1) \mathrm{m}$ like ocean waves, but will be of limited use for assessing rain effects on short laboratory waves.

We note here that the theory of Le Méhauté \& Khangaonkar (1990) also relies on inviscid irrotational wave solutions. Therefore it cannot properly account for waveturbulence interaction and is thus also limited to waves with wavelength $\lambda>O(1) \mathrm{m}$. We believe that to be the source of the discrepancy, last noted by Peirson et al. (2013), between their theory and the laboratory data (Poon et al. 1992; Tsimplis 1992; Harrison 2012; Peirson et al. 2013).

\subsubsection{Smoothness of the free surface and the validity of the kinetic approach}

As noted in section 2.1, the kinetic description of the rain assumes that the radii of rain drops are much smaller than a macroscopic length scales $d L$. In the case of a heavy rain, drops can have radii as large as several millimetres. Looking at figure 2a for example, we find that $r=3 \mathrm{~mm}$ is a reasonable upper limit on the radius (i.e. the density $n(r)$ of drops is very small for rain drops larger $r>3 \mathrm{~mm}$ ). Villermaux \& Bossa (2009) also showed that large drops spontaneously fragment in free fall conditions leading to an effective upper limit for the drop radius. In fact, Villermaux \& Bossa (2009), using established laboratory results showed that the fragmentation of free falling large drops yields a size distribution similar to that of Marshall \& Palmer (1948). Here, this means that our derivation is correct if $d L$ is at least much larger than approximately $3 \mathrm{~mm}$, but further quantifying this limitation is not straightforward.

However, there is an additional and stronger constraint that has to be taken into account. Indeed, the analysis presented in section 2.2 assumes that the rain impacts an element of surface $d S$ that can be defined by a normal unit vector $\mathbf{n}$. This implies that $d S$ is locally flat and thus smooth. Yet, rain drop impacts generate perturbations on the free surface in the form of large impact craters, stalks and secondary droplets, and capillary ring waves. The impact craters have radii that are on the order of $O(10) r$ and so are the height of the stalks (Prosperetti \& Oguz 1993; Yarin 2005). The amplitude and wavelength of capillary ring waves are expected to scale with $O(1) r$ (Bliven et al. 1997). Thus, we expect that the largest surface perturbations generated by the rain are of order $O(10) r$. Since $d S$ is associated with the macroscopic length scale $d L$ (where $d L$ scales with $d S^{1 / 2}$ ), we infer that $d S$ can be considered smooth when $d L$ is at least of $O(100) r$.

Furthermore, $d S$ is locally flat but the free surface is curved because of the surface waves. Accordingly, we suggest that the surface wave wavelength $\lambda$ is required to be larger than $O(10) d L$. Taking a drop radii as a conservative $r \approx 3 \mathrm{~mm}$, together these estimates imply that our approach is valid for wavelength $\lambda$ longer than $\approx 3 \mathrm{~m}$.

\subsubsection{Wave slope and velocity.}

In estimating $p_{d}=p_{d}^{(0)}+p_{d}^{(1)}$, we chose to keep only linear terms in $a k$ (wave slope) and $a \omega$ (amplitude of the surface velocity), ignoring terms in $(a k)^{2}, a^{2} k \omega$ and $a k(a \omega)^{2}$ 
contained in $\left|\left(\boldsymbol{v}_{d}(r)-\boldsymbol{u}\right) \cdot \mathbf{n}\right|^{2}$ in equation (3.6). The linear theory for surface waves already assumes $a k$ as a small parameter so terms in $(a k)^{2}$ are readily negligible. Assuming $\omega=(g k)^{1 / 2}$ (which will be shown below), we find that both $a^{2} k \omega<a k$ and $a k(a \omega)^{2}<a k$ for $k>g(a k)^{2}$. Thus, taking for example the range of validity of the linear model to be for $a k<0.05$, we estimate that terms in $a^{2} k \omega$ and $a k(a \omega)^{2}$ are negligible for surface waves with wavelength $\lambda<O(250) \mathrm{m}$. We conclude here that the linear assumption combined with neglecting high order rain-induced pressure terms makes this approach valid for surface waves with wavelength $\lambda$ shorter than $\approx 250 \mathrm{~m}$. Concurrently, this leads to a maximum surface velocity $a \omega<O(1) \mathrm{m} \mathrm{s}^{-1}$.

\subsubsection{Radius of impacting drops.}

Here, we explore the assumption that $\left(\boldsymbol{v}_{d}(r)-\boldsymbol{u}\right) \cdot \mathbf{n}<0$ is true for all drop radii. To first order in $a k$, the inequality $\left(\boldsymbol{v}_{d}(r)-\boldsymbol{u}\right) \cdot \mathbf{n}<0$ that characterizes impacting drops reduces to $w_{d}(r)-w<0$ with $w$ the vertical component of the surface velocity (i.e. to first order, the surface is flat and $\mathbf{n}$ is vertical). On the forward side of the surface wave, $w>0$, and since $w_{d}(r)<0$ for every $r$, this inequality is always true: every drop impacts the surface. On the back side of the wave where $w<0$, the inequality reduces to $\left|w_{d}(r)\right|>|w|$. Since $w=-\mathrm{i} a \omega \mathrm{e}^{\mathrm{i}(k x-\omega t)}$, the inequality is satisfied if $\left|w_{d}(r)\right|>a \omega$. We note that $|w|=a \omega$ is a "worst case scenario" which only occurs when the wave phase $(k x-\omega t)= \pm \pi / 2$ : for instance, $w=0$ at the crest and trough of the wave. Given equation (3.14), this leads to the constraint on the radius

$$
r>r_{\min }=\frac{1}{\alpha_{w}}\left|\ln \left(1-\frac{a \omega}{\Upsilon_{w}}\right)\right| \approx\left|\frac{1}{\alpha_{w}} \frac{a \omega}{\Upsilon_{w}}\right| .
$$

In other words, drops with radii less than $r_{\min }$ may not reach the surface, at least not in a manner that is homogeneous in space along the waves. Consequently, the integral (3.6) may be over estimated since it is calculated for $r$ in ]0, $+\infty$ [. Conversely, using $r_{\text {min }}$ leads to a slight underestimate of (3.6):

$$
\tilde{p}_{d}=\int_{r_{m i n}}^{+\infty} m(r) n(r)\left|\left(\boldsymbol{v}_{d}(r)-\boldsymbol{u}\right) \cdot \mathbf{n}\right|^{2} d r .
$$

Thus, the error made on the true pressure is at most

$$
\frac{p_{d}-\tilde{p}_{d}}{p_{d}}=\frac{1}{p_{d}} \int_{0}^{r_{\min }} m(r) n(r)\left|\left(\boldsymbol{v}_{d}(r)-\boldsymbol{u}\right) \cdot \mathbf{n}\right|^{2} d r .
$$

Taking $a \omega$ to be at most $O(1) \mathrm{m} \mathrm{s}^{-1}$ as described above, yields $r_{\min } \leqslant 10^{-4} \mathrm{~m}$. And using $n(r)$ and $w_{d}(r)$ in equation (3.18) above, we find an error around $10^{-4 \%}$. This is clearly very small and is due to the fact that the integrand in (3.6) depends on the $r^{3}$ and $w_{d}^{2}$, both of which are small for $r \leqslant r_{\min }$.

\section{Results}

Using the size and velocity distributions for the rain drops given in section 3.2, equations (3.11) and (3.12) can be evaluated in order to assess the effect of rainfall on the propagating surface waves. We note here that the distributions chosen allow for the derivation of an analytical solution (see appendix A), but in general equations (3.11) and (3.12) can easily be estimated numerically. As mentioned above, the specific details of the distributions do not dramatically alter the results.

The first notable result is that for all practical purposes, $\omega_{R} \approx(k g)^{1 / 2}$. This stems from the fact that the terms $\left(k^{2} J^{2}\right) /\left(4 \rho^{2}\right)$ and $\left(k^{4} I^{2}\right) /\left(\rho^{2}\right)$ in equation (3.11) are generally 
much smaller than $(\mathrm{kg})$. The exact same terms appear in equation (3.12) but the two $(\mathrm{kg})$ terms appear with opposite signs, and

$$
\omega_{I} \approx \frac{1}{2 \rho}\left(k J+\frac{k^{2} I}{\omega_{R}}\right) .
$$

In turn, this means that $\omega_{I} \ll \omega_{R}$ and thus, the vertical speed of the free surface due to the wave amplification or attenuation, $a \omega_{I}$, is very small compared to either the surface wave phase speed $(g / k)^{1 / 2}$ or the velocity of the free surface due to the wave propagation, $a \omega_{R}$.

As a side note, it is worth mentioning here that approximation (4.1) can be quickly obtained from a result originally mentioned in Lamb (1932) (Sec. 348) where he estimated the wave attenuation rate

$$
\omega_{I}=\frac{C k}{2 a \rho \omega_{R}},
$$

when the surface is exposed to a wave coherent pressure $p=\mathrm{i} C \mathrm{e}^{\mathrm{i}(k x-\omega t)}$ in quadrature with the surface elevation. Here, from equation (3.7),

$$
C=(I k+J \omega) a,
$$

and it follows that

$$
\begin{aligned}
\omega_{I} & =\frac{C k}{2 a \rho \omega_{R}} \\
& =\frac{k}{2 \rho \omega_{R}}(I k+J \omega) \\
& =\frac{1}{2 \rho}\left(k J+\frac{k^{2} I}{\omega_{R}}\right) .
\end{aligned}
$$

The derivation of Lamb (1932) results from a boundary-layer approximation, assuming that viscous effects near the surface do not significantly alter the inviscid, irrotational wave solution. This is consistent with $\omega_{R} \approx(g k)^{1 / 2}$ and additionally requires that the boundary layer thickness be small compared to the surface wave wavelength, i.e. that $\omega_{I} \ll \omega_{R}$.

In our general case, relatively simple interpretations of $I$ and $J$ can also be given. For example, as noted earlier, $J$ is the rain induced mass flux driven by the vertical velocity, and hence a flux through an horizontal surface. The rain induced mass flux is thus related to the rain rate and $R=J /\left(2 \rho_{d}\right)$. The term containing $J$ appears in equation (3.7) as a pressure involving the vertical velocity of the interface and that of the rain. It is related to the modulation of the momentum flux from rain vertical velocity, locally along the surface wave, by the vertical velocity of the moving surface. In other words, this contribution would vanish if the surface were non-moving. In addition, this term will always have the effect of damping the waves. The integral $I$ is the only term containing the horizontal component of the rain velocity $u_{d}$. It is related to the modulation of the momentum flux from the rain vertical velocity by the rain horizontal velocity and the surface slope $a k$. Therefore, it contains information regarding the direction of the wind compared to that of the surface waves. Here we have chosen waves with $\omega_{R}>0$ propagating in the $x>0$ direction, so $u_{d}<0$ implies $I<0$ meaning that the wind travels opposite to the wave. Respectively, $I>0$ indicates wind and waves propagating in the same direction. The term containing $I$ appears in equation (3.7) as a pressure term involving the surface slope. This term would vanish for a flat interface.

Figure 3a shows the rate of change $\omega_{I} /|R|$ of the surface wave amplitude normalized by 

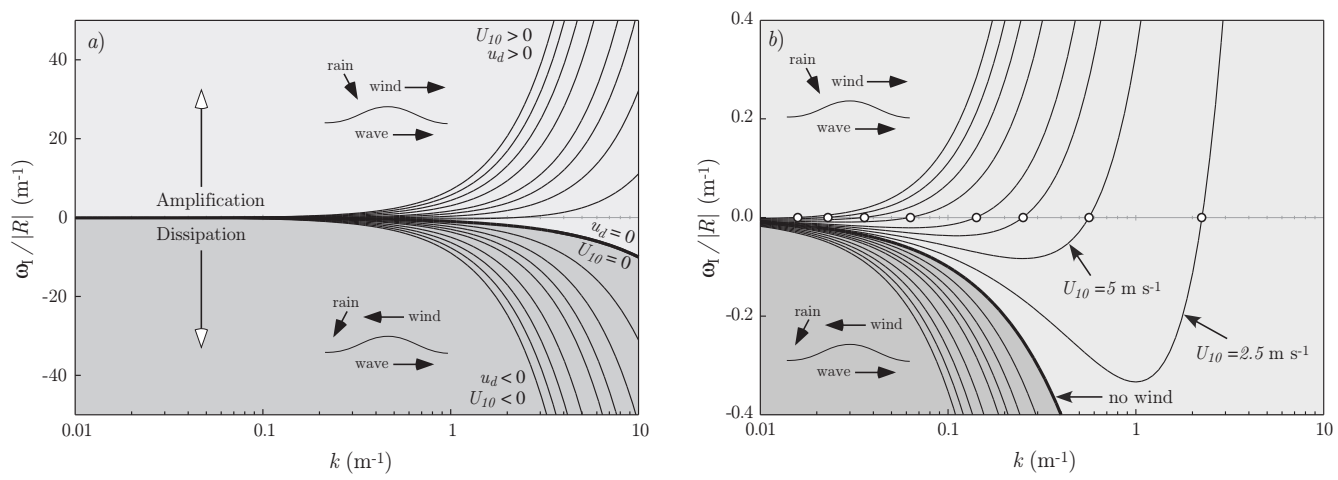

FiguRE 3. a) The rate of change $\omega_{I} /|R|$ of the surface wave amplitude normalized by the absolute value of the rain rate (in $\mathrm{m} \mathrm{s}^{-1}$ ) as a function of the wavenumber $k$ plotted for several wind speeds. The wind direction compared to that of the wave is displayed with the light $\left(U_{10}>0\right)$ and dark $\left(U_{10}<0\right)$ shadings. The thick black line shows $U_{10}=0$ (or $\left.u_{d}=0\right)$. b) shows, for the wind speed studied here, a closer view at the transition from dissipation to amplification at fixed wavenumbers.

the absolute value of the rain rate $\left(\right.$ in $\mathrm{m} \mathrm{s}^{-1}$ ) as a function of the wavenumber $k$ plotted for $10-\mathrm{m}$ equivalent wind speeds of $U_{10}= \pm 0,2.5,5,7.5,10,15,20,25$, and $30 \mathrm{~m}$ $\mathrm{s}^{-1}$. The direction of the wind compared to that of the waves is displayed with light and dark shadings and the schematic embedded in the figure. The thick line shows $u_{d}=0$ (or $U_{10}=0$ ). When $I<0, u_{d}<0$, the wind propagates in the direction opposite to the waves, and $\omega_{I}$ is always negative, meaning that the surface waves are always damped. This is not surprising since, in this case, rainfall will invariably fall with a velocity that is opposite to that of the waves. This is presumably uncommon and would only occur in the situation of swell waves propagating in a different direction than the local wind and wind-driven seas.

On the other hand, when $I>0$ and $u_{d}>0$, the wind and the waves (and the rain drops transported by the wind) all travel in the same direction. In this case, $\omega_{I}$ can be either positive or negative, a result in qualitative agreement with that of Le Méhauté \& Khangaonkar (1990). Figure 3b shows a closer look at the transition from dissipation to amplification. Here, we find that when $I>0$, then $\omega_{I}>0$ for $k I^{2}>g J^{2}$ and the waves are amplified; alternatively, $\omega_{I}<0$ when $k I^{2}<g J^{2}$, and the waves are damped.

The transition from dissipation to amplification occurs at a wavenumber $k_{o}$ such that $k_{o} I^{2}=g J^{2}$ or $c_{o}=\left(g / k_{o}\right)^{1 / 2}=I / J$, i.e. when the ratio $I / J$ matches the phase speed of the surface waves. Figure 4 shows the transition wavenumber $k_{o}$ as a function of $U_{10}$. The open symbols mark the 8 different (non-zero) wind speeds plotted on figure 3 . Wave amplification occurs for wavenumbers $k>k_{o}$ (short waves) and wave dissipation for $k<k_{o}$ (long waves). Also, as a reminder, we show in the shaded areas on figure 4 the range of wavenumber for which the theory presented here starts to become void.

Finally, in the case where $I=0$, that is $u_{d}=0$ and $U_{10}=0$, when the rain falls vertically because of the absence of wind, we find that $\omega_{I}=k J /(2 \rho)$ and $\omega_{I}<0$. Thus, the surface waves are always dissipated and $\omega_{I}$ further reduces to $\omega_{I}=\left(\rho_{d} k R\right) / \rho$, a result previously alluded to by Phillips (1987) who identified the proportionality $\omega_{I} \propto k R$ but did not elaborate further. Here we estimate that with $U_{10}=0 \mathrm{~m} \mathrm{~s}^{-1}$ and with a rain rate of $|R|=50 \mathrm{~mm} \mathrm{~h}^{-1}$, the amplitude of a surface wave of wavelength $\lambda=5 \mathrm{~m}$ would be damped $94 \%$ after 1 hour, $88 \%$ after 2 hours, and $73 \%$ after 5 hours, with a corresponding reduction in wave energy density of $12 \%, 22 \%$, and $46 \%$ respectively. 


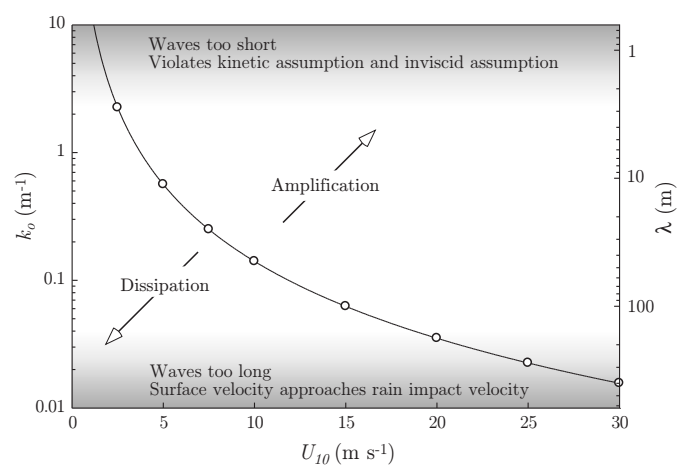

FIgURE 4. Transition wavenumber $k_{o}$ between wave amplification and dissipation as a function of wind speed for $U_{10}>0$. The right vertical axis shows the corresponding surface wave wavelength. We also show the range of validity of the theoretical approach presented here.

\section{Discussion}

\subsection{Comparison with the theory of Le Méhauté \& Khangaonkar (1990)}

As mentioned above, Le Méhauté \& Khangaonkar (1990) modelled rain-wave interactions based only on the rain drop's momentum change at the air-sea interface. They did not account for a distribution of rain drop size or fall velocities and as such, their results depend solely on a rain rate and a unique rain fall velocity. For deep water linear waves, they found that

$$
\omega_{I}=\frac{\rho_{d}|R|}{2 \rho}\left(\frac{U_{d} k^{2}}{\omega_{R}}-3 k\right)
$$

where $U_{d}$ is the rain horizontal fall velocity. However, we find several issues with their derivation. The main problem has to do with their estimate of the modification of the rain rate, locally, when waves are present (their equation (3)). They correctly included the effect of the movement of the surface on the exchange of momentum at the surface but they mistakenly omitted the effect of the surface slope on the mass flux across the surface. In other words, in their version of equation (2.3), they used the vertical component of $(\boldsymbol{v}-\boldsymbol{u})$ instead of $(\boldsymbol{v}-\boldsymbol{u}) \cdot \mathbf{n}$. In addition, they included the effects of the rain-induced (viscous) tangential stress at the surface by accounting for the weight of the viscous boundary layer as a pressure term that was incorporated in the inviscid Euler model. We find that including viscous effects in inviscid models using these boundary layer assumptions can be misleading. In this paper, we limit our analysis to strictly inviscid boundary conditions. Accounting for both these issues (correcting for the sloped surface and eliminating the effects of the shear stress), their corrected formula is:

$$
\omega_{I}=\frac{\rho_{d} R}{2 \rho}\left(2 k-\frac{2 U_{d} k^{2}}{\omega_{R}}\right) .
$$

The similarity between equations (5.2) and (4.1) becomes easily apparent. In fact, as already mentioned, $J=2 \rho_{d} R$. Furthermore, if the drop size distribution $n(r)$ is taken to be monodisperse (as Le Méhauté \& Khangaonkar (1990) do), that is if we greatly simplify $n(r)=\delta_{r-r_{d}}$, then $I$ in equation (4.1) reduces exactly to $I=-2 \rho_{d} R U_{d}$, and equations (5.2) and (4.1) become identical. We note that in this simplified case, the transition from amplification to attenuation arises when $c_{o}=U_{d}$ and the rain will amplify (resp. attenuate) the wave when the horizontal impact velocity is larger (resp. smaller) than the wave phase speed.

In short, in this application to rain falling on surface waves where the distribution 
function $f(t, \boldsymbol{x}, \boldsymbol{v}, r)$ is replaced by a rather simple homogeneous and monokinetic expression, the kinetic approach presented here can be viewed as an extension of the work of Le Méhauté \& Khangaonkar (1990) which allows to retain the dependence of the rain-induced momentum flux on the velocity and size of the drops. Of course, when using a general distribution $f(t, \boldsymbol{x}, \boldsymbol{v}, r)$, the kinetic theory applies broadly to drop momentum flux on moving surfaces (see equation (2.6)) with the possibility to consider non-homogeneous particle distributions, multivalued velocity distributions for a given radius, or include other drop specific parameters such as temperature.

\subsection{Comparison with experimental data}

At this point, it is difficult to compare our theoretical estimates with available data (Manton 1973; Tsimplis \& Thorpe 1989; Tsimplis 1992; Poon et al. 1992; Yang et al. 1997; Braun et al. 2002; Harrison 2012; Peirson et al. 2013) because the experiments on wave-rain interactions all use laboratory, short wind-generated or mechanical waves which systematically fall outside the wavenumber range for which the we consider the present theory to be robust (see figure 5). . We also note that the laboratory observations show weak dependence of the wave attenuation rate on the rain rate, at least at high rain rates. This result is also in contrast with the theory presented here or that of Le Méhauté \& Khangaonkar (1990). Although, Peirson et al. (2013) compared the time scales between consecutive drop impact with that of the lifetime of surface perturbations and concluded that, beyond approximately $|R|=30 \mathrm{~mm} \mathrm{~h}^{-1}$, rain likely falls on a highly perturbed surface which may lead to a "saturated" regime. Figure 5 shows the non-dimensional wave amplitude attenuation rate $-\omega_{I} / \omega_{R}$ (thick black line) as a function of the surface wave wavenumber in the case of a vertical rain (no wind) with $|R|=100$. We also plot the results of Teixeira \& Belcher (2002) who estimated wave damping by sub-surface turbulence using rapid distortion theory. Here we have used a sub-surface turbulence of $u_{r m s}^{\prime}=1.2 \mathrm{~cm} \mathrm{~s}^{-1}$ as did Peirson et al. (2013). Coincidentally, this corresponds to rain induced turbulent levels observed at the surface by Harrison (2012). Teixeira \& Belcher (2002) estimated their results to be valid for $5 \mathrm{~cm} \ll \lambda \ll 15 \mathrm{~m}$ (with $a k=0.1$ and $u_{r m s}^{\prime} \approx 1 \mathrm{~cm} \mathrm{~s}^{-1}$ ). The data of Tsimplis (1992); Harrison (2012); Peirson et al. (2013) are shown with the symbols. The data show significant scatter and a trend is difficult to extract. Despite the scatter in the data however, it is also clear that a simple linear extrapolation of the kinetic theory beyond the range of validity suggested above in section 3.3 is not recommended.

The subsurface turbulence measurements of Harrison (2012) and Peirson et al. (2013) show that the turbulent motions under the rain are surprisingly weak and also independent of rain rate. Note here that with turbulent levels independent of rain rate, the viscous damping of the waves by the turbulence would also be independent of rain rate as is indeed observed in the laboratory (Poon et al. 1992; Tsimplis 1992; Harrison 2012). Peirson et al. (2013) concluded that high levels of dissipation must be present at the interface and that near-saturated turbulent conditions exist at high rain rates. They hypothesize that below a threshold rain rate, of $O(30) \mathrm{mm} \mathrm{h}^{-1}$, rain-rate-sensitive regimes may exist. However, our current understanding of turbulence generated at the surface and its interaction with short waves is considerably incomplete and more work in the area is needed (Poon et al. 1992; Peirson et al. 2013).

In any case, as mentioned earlier, the thin rain-induced turbulent layer only interacts with waves shorter than $O(1) \mathrm{m}$ and viscous damping of these short waves by the turbulence leads to $\omega_{I} \propto k^{2}$. The approach presented here applies for waves longer than $O(1-3) \mathrm{m}$ and under vertical rain conditions where $I=0$, the present model yields $\omega_{I} \propto k$. Thus, technically, it should be possible to perform simple experiments that would 


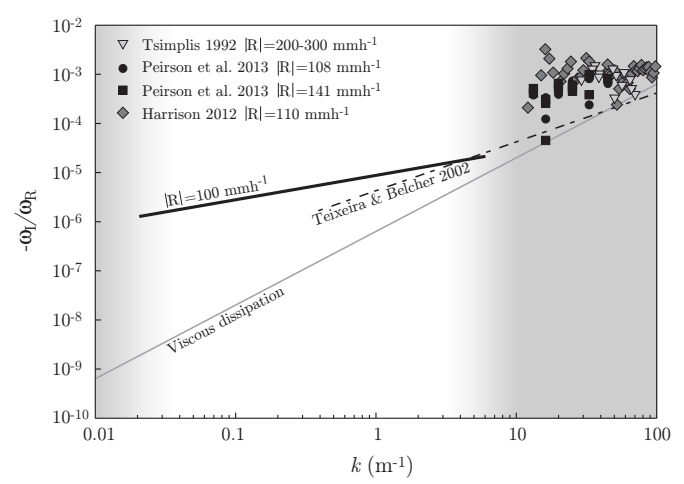

Figure 5. Non-dimensional attenuation rate as a function of the surface wave wavenumber in the case of vertical rain (no wind) with a rain rate of $|R|=100 \mathrm{~mm} \mathrm{~h}^{-1}$ (thick black line). The dash line shows the the estimate of Teixeira \& Belcher (2002) from rapid distortion theory using a subsurface turbulence of $u_{r m s}^{\prime}=1.2 \mathrm{~cm} \mathrm{~s}^{-1}$. The data symbols show the data of Tsimplis (1992); Harrison (2012); Peirson et al. (2013). The solid gray line shows viscous attenuation. As in figure 4 , the gray area shows the range over which the present theory becomes void.

span a sufficiently large wavenumber range to evaluate both regimes. We note here that the data of Peirson et al. (2013) suggest $\omega_{I} \propto k^{3 / 2}$. Besides experiments, improvement to the theoretical approach can also be considered. To this end, we intend to use the rain stress formulation developed here with a typical rain distribution and include it in a direct Navier-Stokes simulation in the hope that the blended approach might be able to account for the effects of the turbulence on the short waves.

Furthermore, with our kinetic approach it is also possible, in principle, to include other effects like mass and heat transfer, and to take into account the difference in density (salinity) between rain drops and sea water. This last property might be very important for wave attenuation as it induces stratification in the surface layer which in turn constrains the propagation of the vortices generated by drop impacts and affects the structure of the near surface turbulence (Harrison 2012).

In practice, it is likely that the kinetic approach as presented here is adequate for "long" waves, but not for the short waves found in the laboratory. At these short scales, subsurface turbulence and wave-turbulence interaction need to be examined and perhaps accounted for in more detail.

\section{Conclusion}

In this paper, we present a kinetic model for estimating the momentum flux from falling drops onto a moving free surface. This approach neglects the internal structure of the particles, but retains other properties, like their radius and velocity, and hence their momentum. The model also takes into account the disperse nature of particles in size, position, and velocity, through a time-dependent distribution function $f(t, \boldsymbol{x}, \boldsymbol{v}, r)$. The computation of the stress exerted by the particles on a moving free surface then becomes straightforward and is easily incorporated in Navier-Stokes and Euler free surface models.

As an example of a possible application of our approach, we apply the present model to the problem of rain falling on linear deep water surface waves. In this particular case, $f(t, \boldsymbol{x}, \boldsymbol{v}, r)$ is replaced by a rather simple expression. Under appropriate linear, inviscid, and irrotational conditions, the rain-induced stress is included in the free surface Euler model as a wave-coherent surface pressure. The kinetic approach allows us to retain the 
dependence of the rain-induced momentum flux on the velocity and size of the drops. In addition, we show that the rain stress also depends on the free surface velocity and slope.

For monochromatic linear surface waves, we find that our model is valid for surface wavelengths approximately ranging from $3 \mathrm{~m}$ to $250 \mathrm{~m}$. Our results show that rainfall can induce either wave amplification or attenuation depending on the wind speed and direction, and the surface wave wavelength. For example, in the case of vertical falling rain, in the absence of wind, waves appear to be attenuated over the whole wavenumber range and the attenuation rate reduces to a linear function of the rain rate and surface wave wavenumber. In general, our theory can be viewed as an extension of the work Le Méhauté \& Khangaonkar (1990) who considered a monodipserse rain size distribution. In the work presented here, the attenuation rate is related to moments of $f(t, \boldsymbol{x}, \boldsymbol{v}, r)$.

Acknowledgments. This study has been carried out in the frame of the Investments for the future Programme IdEx Bordeaux CPU (ANR-10-IDEX-03-02). We wish to thank several anonymous reviewers whose comments and suggestions helped improve the paper.

\section{Appendix A}

As mentioned in section 3, several rain drop size distributions exist in the literature. The most common drop size distributions are variations of the modified gamma distribution (Ulbrich 1983):

$$
n(r)=\mathcal{N} r^{k} e^{-\Lambda r},
$$

with $\mathcal{N}$ a normalization factor. Generally, both $\Lambda$ and $\mathcal{N}$ depend on the rain rate to account, for example, for the change in the shape of the distribution with a shift to larger drop under intense rain events. We also note that the normalization factor $\mathcal{N}$ essentially allows for the rain rate to be recovered such that:

$$
\begin{aligned}
R & =\int_{] 0,+\infty[} \frac{m(r)}{\rho_{d}} n(r) w_{d}(r) d r \\
& =\mathcal{N} \int_{] 0,+\infty[} \frac{m(r)}{\rho_{d}} r^{k} e^{-\Lambda r} w_{d}(r) d r .
\end{aligned}
$$

As such, $\mathcal{N}$ also depends on the choice of the radius-dependent vertical velocity $w_{d}(r)$.

When integral properties of the rain size distributions, as is the rain rate $R$ for example, are the only variables needed, it is sometimes possible to evaluate these integrals analytically. For example, Schlussel et al. (1997) used the distribution (A 1) with $k=0$ originally proposed by Marshall \& Palmer (1948) and provided analytical expression for the fresh water input by rainfall to the upper layers of the ocean. In our case, we need to evaluate the equations (3.8) in which both horizontal $u_{d}(r)$ and vertical $w_{d}(r)$ components of the impact drop velocity also appears. To do so, we too choose the drop size distribution proposed by Marshall \& Palmer (1948):

$$
n(r)=\mathcal{N} e^{-\Lambda r},
$$

along with the following formulations for impact drop velocity components:

$$
\begin{aligned}
& u_{d}(r)=\Upsilon_{u}\left(1-\mathrm{e}^{-\alpha_{u} r}\right), \\
& w_{d}(r)=-\Upsilon_{w}\left(1-\mathrm{e}^{-\alpha_{w} r}\right) .
\end{aligned}
$$

Under these conditions, and using the rain rate $R$ as the independent variable, the 
normalized drop size distribution is:

$$
n(r)=\frac{R e^{-\Lambda r}}{8 \pi \Upsilon_{w}}\left(\frac{1}{\left(\Lambda+\alpha_{w}\right)^{4}}-\frac{1}{\Lambda^{4}}\right)^{-1},
$$

which indeed depends on the details of the vertical fall velocity. Additionally, the equations (3.8) reduce to:

$$
\begin{aligned}
& I=2 \rho_{d} R \Upsilon_{u}\left(\frac{\left(\Lambda+\alpha_{w}+\alpha_{u}\right)^{-4}-\left(\Lambda+\alpha_{u}\right)^{-4}}{\left(\Lambda+\alpha_{w}\right)^{-4}-(\Lambda)^{-4}}-1\right), \\
& J=2 \rho_{d} R .
\end{aligned}
$$

Values for $\Lambda, \Upsilon_{u}, \alpha_{u}, \Upsilon_{w}$, and $\alpha_{w}$ are cited in the text or can be chosen from sources in the literature.

\section{REFERENCES}

AtLAs, D 1994 Footprints of storms on the sea: A view from spaceborne synthetic aperture radar. J. Geophys. Res. 99 (C4), 7961-7969.

BEst, AC 1950 Empirical formulae for the terminal velocity of water drops falling through the atmosphere. Q. J. R. Meteorol. Soc. 76, 302-311.

Beya, J, Peirson, W \& Banner, M 2011 Rainfall-generated, near-surface turbulence. In Gas Transfers at Water Surfaces 2010, S. Komori, W. McGillis and R. Kurose eds., Kyoto, pp. 90-103. University Press.

Bliven, L, Branger, H, Sobieski, P \& Giovanangeli, JP 1993 An analysis of scatterometer returns from a water agitated by artificial rain. International Journal of Remote Sensing 14 (12), 2315-2329.

Bliven, L F, Sobieski, P W \& Craeye, C 1997 Rain generated ring-waves: measurements and modelling for remote sensing. International Journal of Remote Sensing 18 (1), 221-228.

Braun, N 2003 Untersuchungen zur radar-rückstreuung und wellendämpfung beregneter wasseroberflächen, dissertation, universität hamburg, fachbereich geowissenschaften, on the radar backscattering and wave damping on water surfaces agitated by rain. Phd dissertation, University of Hamburg.

Braun, N, Gade, M \& LAnge, P A 2002 The effect of artificial rain on wave spectra and multipolarisation x band radar backscatter. International Journal of Remote Sensing 23 (20), 4305-4323.

Caldwell, D R \& Elliott, W P 1971 Surface stresses produced by rainfall. J. Phys. Oceanogr. $1(2), 145-148$.

Caldwell, D R \& Elliott, W P 1972 The effect of rainfall on the wind in the surface layer. Boundary-Layer Meteorology 3 (2), 146-151.

Chapman, S \& Cowling, TG 1990 The mathematical theory of nonuniform gases, 3rd edn. Cambridge University Press, Cambridge.

Chen, G, Chapron, B, Tournadre, J, Katsaros, K \& Vandemark, D 1998 Identification of possible wave damping by rain using topex and tmr data. Remote Sensing of Environment 63 (1), 40-48.

Contreras, R F, Plant, W J, Keller, W C, Hayes, K \& Nystuen, J 2003 Effects of rain on ku-band backscatter from the ocean. Journal of Geophysical Research-Oceans 108 (C5), 3165.

Csanady, G T 2001 Air-Sea Interaction Laws and Mechanisms. Cambridge University Press.

Green, T \& Houk, D F 1979b The mixing of rain with near-surface water. J. Fluid Mech. 90, $569-588$.

Guymer, T H, Quartly, G D \& Srokosz, M A 1995 The effects of rain on ers-1 radar altimeter data. J. Atmos. Oceanic Technol. 12, 1229-1247.

HARRISON, E L 2012 The effects of rainfall in the ocean surface at low to moderate wind speed. Phd dissertation, University of Delaware.

Harrison, E L, Veron, F, Ho, D T, Reid, M S, Orton, P \& McGillis, W R 2012 
Nonlinear interaction between rain- and wind-induced air-water gas exchange. J. Geophys. Res. 117 (C03034).

Ho, D T, Asher, W E, Bliven, L F, Schlosser, P \& Gordan, E L 2000 On mechanisms of rain-induced air-water gas exchange. Journal of Geophysical Research-Oceans 105 (C10), 24045-24057.

Jones, ISF \& ToBa, Y 2001 Wind stress over the ocean. Cambridge University Press.

Kraus, E B \& Bussinger, JA 1994 Atmosphere-Ocean Interaction. Oxford University Press. LAMB, H 1932 Hydrodynamics. (Sixth Ed.), Cambridge university press.

Law, JO \& Parsons, DA 1943 The relation of raindrop-size intensity. Trans. Amer. Geophys. Union 24, 452-460.

Le Méhauté, B \& Khangaonkar, T 1990 Dynamic interaction of intense rain with water waves. Journal of Physical Oceanography 20 (12), 1805-1812.

Manton, M J 1973 On the attenuation of sea waves by rain. Geophysical and Astrophysical Fluid Dynamics 5, 249-260.

Marchisio, E \& Fox, RO 2013 Computational Models for Polydisperse Particulate and Multiphase Systems. Cambridge University Press.

Marshall, JS \& PALmer, WMK. 1948 The distribution of raindrops with size. J. Meteor. 5, $165-166$.

Melville, W K 1996 The role of surface-wave breaking in air-sea interaction. Annu. Rev. Fluid Mech. 28, 279-321.

Mueller, J A \& Veron, F 2009 A lagrangian stochastic model for heavy particle dispersion in the atmospheric marine boundary layer. Bound.-Layer Meteor. 130 (2), 229-247.

Nystuen, J A 1990 A note on the attenuation of surface gravity waves by rainfall. Journal of Geophysical Research-Oceans 95 (C10), 18353-18355.

Peirson, WL, Beya, JF, Banner, ML, Peral, JS \& Azarmsa, SA 2013 Rain-induced attenuation of deep-water waves. J. Fluid Mech. 724, 5-35.

PhILlips, O M 1987 Ocean-wave predictions: where are we? Johns Hopkins U. APL Technical Digest 8 (1), 7-10.

Poon, Y K, TANG, S \& Wu, J 1992 Interactions between rain and wind waves. Journal of Physical Oceanography 22 (9), 976-987.

Prosperetti, A \& Oguz, H N 1993 The impact of drops on liquid surfaces and the underwater noise of rain. Annu. Rev. Fluid Mech. 25, 577-602.

Quartly, G D, Guymer, T H \& Srokosz, M A 1996 The effects of rain on topex radar altimeter data. J. Atmos. Oceanic Technol. 13, 12091229.

Reynolds, O 1900 On the action of rain to calm the sea, Papers on Mechanical and Physical Subject, 1870-1880 Collected Works, vol. 1, pp. 86-88. Cambridge: Cambridge University Press.

Schlussel, P, Soloviev, A V \& Emery, W J 1997 Cool and freshwater skin of the ocean during rainfall. Boundary-Layer Meteorology 82 (3), 439-474.

Sullivan, P P \& MCWilliams, J C 2010 Dynamics of winds and currents coupled to surface waves. Annu. Rev. Fluid Mech. 42, 19-42.

Teixeira, M A C \& Belcher, S E 2002 On the distortion of turbulence by a progressive surface wave. J. Fluid Mech. 458, 229-267.

Thorpe, S A 2004 Langmuir circulation. Annu. Rev. Fluid Mech. 36, 55-79.

Tsimplis, M \& Thorpe, S A 1989 Wave damping by rain. Nature 342, 893-895.

Tsimplis, M N 1992 The effect of rain in calming the sea. Journal of Physical Oceanography 22 (4), 404-412.

Ulbrich, C W 1983 Natural variations in the analytical form of the raindrop size distribution. J. Climate Appl. Meteor. 22, 1764-1775.

Veron, F 2015 Ocean spray. Annu. Rev. Fluid Mech. 47, 507-538.

Villermaux, E \& Bossa, B 2009 Single-drop fragmentation distribution of raindrops. Nature Physics 5 (9).

Weissman, D E, Stiles, BW, Hristova-Veleva, SM, Long, DG, Smith, DK, Hilburn, KA \& JONES, LW 2012 Challenges to satellite sensors of ocean winds: Addressing precipitation effects. Journal of Atmospheric and Oceanic Technology 29, 356374. 
DE Wolf, D A 2001 On the laws-parsons distribution of raindrop sizes. Radio Science 36 (4), 639-642.

YANG, Z, TANG, S \& Wu, J 1997 An experimental study of rain effects on fine structures of wind waves. Journal of Physical Oceanography 27 (3), 419-430.

YARIN, A L 2005 Drop impact dynamics: splashing, spreading, receding, bouncing. Annu. Rev. Fluid Mech. 38, 159-192. 